\title{
MODELLING THE EFFECT OF ICONICITY ON ITERATED (CROSS-SITUATIONAL) LEARNING
}

\author{
Bart de Boer ${ }^{1}$ \\ bart@ai.vub.ac.be \\ ${ }^{1}$ AI-lab, Vrije Universiteit Brussel, Brussels, Belgium
}

Iconicity has been shown to have an important influence on both the learnability of lexical items (Dingemanse, Blasi, Lupyan, Christiansen, \& Monaghan, 2015) and on the way in which systems of signals emerge in cultural evolution (Little, Eryllmaz, \& de Boer, 2017; Roberts, Lewandowski, \& Galantucci, 2015). However, the effect of iconicity is different under different circumstances, sometimes facilitating learning, sometimes hindering it (reviewed in Dingemanse et al. 2015). Iconicity may help early acquisition and emergence of language, but when a communication system grows, iconicity may actually become problematic and arbitrary form-meaning mappings may be more advantageous. Thus, iconicity may have played a different role in early evolution of language than it plays today.

Because of the different effects of iconicity under different circumstances (which may not all be controllable in experiments or observational studies) and because its role may have changed during biological evolution, here an agentbased computer model is used to investigate the effect of iconicity on cultural evolution of lexical systems. The model is based on cross-situational learning, where two agents communicate about a situation in which multiple meanings are present; one agent signals a meaning; the other needs to learn which signal corresponds to which meaning. The learning agent needs to recover the correct associations between signals and meanings. The original contribution of this model is that meanings and signals may both trigger identical sensory features, and hence can be iconic. When an agent determines a signal's meaning, it not only uses the learned associations, but also the (innately determined) similarity between the signal and the meanings present. Both signals and meanings are represented by 10 continuous features with a range of $[-4,4]$. They share 5 of these features. 
Preliminary results of applying the model to transmission chains (in which there is one teacher and one learner, and the learner becomes the teacher in the next generation) are given in Table 1 . Success of communication at the $10^{\text {th }}$ generation (convergence takes place rapidly, so that it is nearly always complete at this point) was observed for 1) iconic and non-iconic transmission, where 2) the initial agent was a perfect communicator (i.e. one meaning corresponds to exactly one signal and vice versa) or was initialized randomly, where 3) reproduction made use of sampling or of a-posteriory maximization (i.e. formmeaning mappings were reproduced with the probability by which they were observed, or each meaning was coupled with the most frequently observed signal), and where 4) the probability of each meaning occurring was uniform, or followed a Zipf distribution (i.e. some meanings were much more frequent than others). Lexicons consisted of 20 words, were trained for 10000 interactions, and contexts consisted of 5 meanings. All runs were repeated 50 times with different random seeds. The parameters were set such that learning was neither too complex nor too simple

It can be observed that maximizers work much better than samplers (as was expected from Smith \& Kirby, 2008), that meanings with a Zipf distribution generally fare somewhat better than uniformly distributed meanings and that maximizers manage to transmit systems quite well, and even to create them from scratch (i.e. in the case of random initializations). However, the effect of iconicity in maximizers appears very marginal, if there is an effect at all. There is a clear effect in samplers, but they do worse than maximizers in all cases.

It is perhaps logical that in transmission chains with one teacher and learner, iconicity does not have a strong influence on success in the case of maximizers: success depends on the fact that the teacher has only one signal associated with each meaning, and the learner needs to learn (and later reproduce) this. Thus, iconicity is only a distraction. However, in a social coordination setting, where multiple agents negotiate a signaling system from scratch, iconicity may play an important role in helping the agents converge to the same signal for the same meaning. Investigating this hypothesis is the next step in this modeling effort, as well as investigating what happens to the influence of iconicity when the lexicon grows and becomes conventionalized. It should also be investigated whether there are any situations in which samplers do better than maximizers.

Table 1. Communicative success in the tenth generation for different configurations of the model. Numbers represent: median $_{1 \text { st quartile }}^{3 \text { rd quatile }}$

\begin{tabular}{|c|c|c|c|c|}
\hline & \multicolumn{4}{|c|}{ Perfect Initialization } \\
\hline & \multicolumn{2}{|c|}{ Sampler } & \multicolumn{2}{|c|}{ Maximizer } \\
\hline & uniform & Zipf & uniform & Zipf \\
\hline not iconic & $0.05_{0.05}^{0.05}$ & $0.17_{0.17}^{0.17}$ & $1_{1}^{1}$ & $1_{1}^{1}$ \\
\hline iconic & $0.39_{0.33}^{0.47}$ & $0.36_{0.31}^{0.39}$ & $1_{1}^{1}$ & $0.97_{0.95}^{1.00}$ \\
\hline
\end{tabular}


Random Initialization

\begin{tabular}{|c|c|c|c|c|}
\hline \multirow[b]{2}{*}{ not iconic } & & & & \\
\hline & $0.05_{0.05}^{0.05}$ & $0.17_{0.17}^{0.17}$ & $0.70_{0.70}^{0.75}$ & $0.86_{0.83}^{0.90}$ \\
\hline iconic & $0.41_{0.34}^{0.48}$ & $0.35_{0.29}^{0.38}$ & $0.75_{0.70}^{0.80}$ & $0.85_{0.83}^{0.89}$ \\
\hline
\end{tabular}

\section{References}

Dingemanse, M., Blasi, D. E., Lupyan, G., Christiansen, M. H., \& Monaghan, P. (2015). Arbitrariness, Iconicity, and Systematicity in Language. Trends in Cognitive Sciences, 19(10), 603-615.

Little, H., Eryılmaz, K., \& de Boer, B. (2017). Signal dimensionality and the emergence of combinatorial structure. Cognition, 168(1), 1-15.

Roberts, G., Lewandowski, J., \& Galantucci, B. (2015). How communication changes when we cannot mime the world: Experimental evidence for the effect of iconicity on combinatoriality. Cognition, 141, 52-66.

Smith, K., \& Kirby, S. (2008). Cultural evolution: implications for understanding the human language faculty and its evolution. Philosophical Transactions of the Royal Society of London B, 363, 3591-3603. 